TRANSACTIONS OF THE

AMERICAN MATHEMATICAL SOCIETY

Volume 359, Number 2, February 2007, Pages 671-686

S 0002-9947(06)03906-7

Article electronically published on July 20, 2006

\title{
GENERALIZED AHLFORS FUNCTIONS
}

\author{
MIRAN ČERNE AND MANUEL FLORES
}

\begin{abstract}
Let $\Sigma$ be a bordered Riemann surface with genus $g$ and $m$ boundary components. Let $\left\{\gamma_{z}\right\}_{z \in \partial \Sigma}$ be a smooth family of smooth Jordan curves in $\mathbb{C}$ which all contain the point 0 in their interior. Let $p \in \Sigma$ and let $\mathcal{F}$ be the family of all bounded holomorphic functions $f$ on $\Sigma$ such that $f(p) \geq 0$ and $f(z) \in \widehat{\gamma_{z}}$ for almost every $z \in \partial \Sigma$. Then there exists a smooth up to the boundary holomorphic function $f_{0} \in \mathcal{F}$ with at most $2 g+m-1$ zeros on $\Sigma$ so that $f_{0}(z) \in \gamma_{z}$ for every $z \in \partial \Sigma$ and such that $f_{0}(p) \geq f(p)$ for every $f \in \mathcal{F}$. If, in addition, all the curves $\left\{\gamma_{z}\right\}_{z \in \partial \Sigma}$ are strictly convex, then $f_{0}$ is unique among all the functions from the family $\mathcal{F}$.
\end{abstract}

\section{INTRODUCTION}

Let $\Sigma$ be the interior of a bordered Riemann surface with genus $g$ and $m$ real analytic boundary components. Let $p \in \Sigma$ and let $\mathcal{F}_{p}$ be the family of all holomorphic functions from $\Sigma$ to the unit disc $\Delta$ which take $p$ to 0 and which have, in a fixed coordinate chart, nonnegative derivative at $p$. Trying to imitate the proof of the Riemann mapping theorem, that is, trying to maximize the derivative $f^{\prime}(p)$ over all functions $f \in \mathcal{F}_{p}$, one gets the Ahlfors function at point $p$ [1, 2]. The existence of the 'maximal' function follows from the normal family argument, however it is nontrivial to show that any Ahlfors function is a proper map from $\Sigma$ to $\Delta$ and that it has at most $2 g+m$ zeros on $\Sigma$, 1, 2, For importance and usefulness of Ahlfors functions see [6, 7, 8, 9, 13, 14.

In this paper we give some natural generalizations of these results which we hope will give some new insight on the geometry of the problem. We will say that a family of simple closed curves $\left\{\gamma_{z}\right\}_{z \in \partial \Sigma}$ in $\mathbb{C}$ is a $C^{k}(k \in \mathbb{N})$ family of Jordan curves in $\mathbb{C}$ if there exists a function $\rho \in C^{k}(\partial \Sigma \times \mathbb{C})$ such that

$$
\gamma_{z}=\{w \in \mathbb{C} ; \rho(z, w)=0\}
$$

and $\left(\bar{\partial}_{w} \rho\right)(z, w) \neq 0$ for every $z \in \partial \Sigma$ and $w \in \gamma_{z}$. We call $\rho$ a defining function for the family $\left\{\gamma_{z}\right\}_{z \in \partial \Sigma}$.

Received by the editors June 21, 2004 and, in revised form, November 22, 2004.

2000 Mathematics Subject Classification. Primary 35Q15; Secondary 32E99, 30E25.

Key words and phrases. Bordered Riemann surface, Ahlfors function, Riemann-Hilbert problem.

The first author was supported in part by a grant "Analiza in geometrija" P1-0291 from the Ministry of Education, Science and Sport of the Republic of Slovenia. Part of this work was done while the author was visiting the University of La Laguna, Tenerife, Spain. He wishes to thank the faculty of the Analysis Department for their hospitality and support.

The second author was supported in part by grants from FEDER y Ministerio de Ciencia y Tecnologia number BFM2001-3894 and Consejeria de Educacion Cultura y Deportes del Gobierno de Canarias, PI 2003/068.

(C)2006 American Mathematical Society Reverts to public domain 28 years from publication 
The interior of a simple closed curve $\gamma \subseteq \mathbb{C}$ is the bounded component of $\mathbb{C} \backslash \gamma$, and $\widehat{\gamma}$ will denote the closure of the interior of $\gamma$. We will say that a simple closed curve $\gamma \subseteq \mathbb{C}$ is convex if $\widehat{\gamma}$ is a convex set in $\mathbb{C}$, and that $\gamma \subseteq \mathbb{C}$ is strictly convex if $\widehat{\gamma}$ is a strictly convex set in $\mathbb{C}$.

Let $k$ be a nonnegative integer and let $0<\alpha<1$. We denote by $C^{k, \alpha}(\partial \Sigma)$ the Hölder space of all real $k$ times differentiable functions on the boundary $\partial \Sigma$ whose derivatives of order $k$ are Hölder continuous of order $\alpha$, and we denote by $A^{k, \alpha}(\Sigma)$ the space of all holomorphic functions on $\Sigma$ which are of class $C^{k, \alpha}$ on $\bar{\Sigma}$.

Let $p \in \Sigma$ and let $\mathcal{F}$ be the family of all bounded holomorphic functions $f$ on $\Sigma$ such that $f(p) \geq 0$ and $f(z) \in \widehat{\gamma_{z}}$ for almost every $z \in \partial \Sigma$.

Theorem 1.1. Let $\Sigma$ be a bordered Riemann surface with genus $g$ and $m$ real analytic boundary components. Let $\left\{\gamma_{z}\right\}_{z \in \partial \Sigma}$ be a $C^{k+1}(k \geq 3)$ family of Jordan curves in $\mathbb{C}$ which all contain the point 0 in their interior. Then there exists a holomorphic function $f_{0} \in \mathcal{F} \cap A^{k, \alpha}(\Sigma)$ with at most $2 g+m-1$ zeros on $\Sigma$ so that $f_{0}$ is a 'proper' map, that is, $f_{0}(z) \in \gamma_{z}$ for every $z \in \partial \Sigma$, and such that $f_{0}(p) \geq f(p)$ for every $f \in \mathcal{F}$.

In the case of the disc $\Sigma=\Delta$ the result follows from results in [20. See also $[29$. In addition, it also follows from results in 20] that $f_{0}$ is unique among all functions from $\mathcal{F}$. Although it seems natural to expect that the same uniqueness holds in general, we were only able to show the uniqueness in the case of the strictly convex curves $\gamma_{z}$.

Theorem 1.2. Let $\left\{\gamma_{z}\right\}_{z \in \partial \Sigma}$ be a $C^{k+1}(k \geq 3)$ family of convex Jordan curves in $\mathbb{C}$ which all contain the point 0 in their interior and which are strictly convex on a set $z \in \partial \Sigma$ with positive measure. Then there is a unique function $f_{0} \in \mathcal{F}$ such that

$$
f_{0}(p)=\max \{f(p) ; f \in \mathcal{F}\} .
$$

To include Ahlfors functions in these results one needs the following corollary.

Corollary 1.3. Let $n \in \mathbb{N}$ and let $\mathcal{D} \geq n$ be a divisor on $\Sigma$ of a finite degree whose coefficient at $p$ is $n$. Let $\mathcal{F}_{\mathcal{D}}$ be the family of all bounded holomorphic functions $f$ on $\Sigma$ such that $(f) \geq \mathcal{D}, f(z) \in \widehat{\gamma_{z}}$ for almost every $z \in \partial \Sigma$ and which have, in a fixed coordinate chart, a nonnegative $n$-th derivative at $p$. Then there exists a holomorphic function $f_{0} \in \mathcal{F}_{\mathcal{D}} \cap A^{k, \alpha}(\Sigma)$ with at most $\operatorname{deg}(\mathcal{D})+2 g+m-1$ zeros on $\Sigma$ so that $f_{0}$ is a 'proper' map, that is, $f_{0}(z) \in \gamma_{z}$ for every $z \in \partial \Sigma$, and such that $f_{0}^{(n)}(p) \geq f^{(n)}(p)$ for every $f \in \mathcal{F}_{\mathcal{D}}$. If, in addition, the family of Jordan curves $\left\{\gamma_{z}\right\}_{z \in \partial \Sigma}$ is convex for every $z \in \partial \Sigma$ and strictly convex on a set $z \in \partial \Sigma$ with positive measure, there is a unique function $f_{0} \in \mathcal{F}_{\mathcal{D}}$ such that

$$
f_{0}^{(n)}(p)=\max \left\{f^{(n)}(p) ; f \in \mathcal{F}_{\mathcal{D}}\right\} .
$$

A consequence of this corollary is also a result from [11, where the following special case was considered: $\Sigma$ is a planar domain $(g=0)$, divisor $\mathcal{D}=n p$ and all the curves $\gamma_{z}(z \in \partial \Sigma)$ are unit circles centered at 0 .

To put these results in a wider context we recall the notion of the (nonlinear) Riemann-Hilbert problem on $\Sigma$, which for a given $C^{k}$ family of simple closed curves $\left\{\gamma_{z}\right\}_{z \in \partial \Sigma}$ in $\mathbb{C}$ asks for a continuous up to the boundary holomorphic function $f$ on $\Sigma$ such that $f(z) \in \gamma_{z}$ for every $z \in \partial \Sigma$. It was proved in [12] that if $k \geq 4$ and if all curves $\gamma_{z}(z \in \partial \Sigma)$ contain the point 0 in their interior, then there exists a solution of the corresponding Riemann-Hilbert problem with at most $2 g+m-1$ zeros on $\Sigma$. 
Similar previous results can be found in [5, 20, 30. See also [17, 18, 19, 25, 31] and the references therein. The condition that all curves $\gamma_{z}(z \in \partial \Sigma)$ contain the point 0 in their interior can be replaced $([12,20])$ by a seemingly more general, however equivalent, condition that there exists a continuous analytic selector for $\left\{\gamma_{z}\right\}_{z \in \partial \Sigma}$, that is, that there exists a continuous up to the boundary holomorphic function $f$ on $\Sigma$ such that $f(z) \in \operatorname{Int}\left(\gamma_{z}\right)$ for every $z \in \partial \Sigma$. Hence it follows that all our results also appropriately hold in the cases where the condition that all curves $\left\{\gamma_{z}\right\}_{z \in \partial \Sigma}$ contain the point 0 in their interior is replaced by the condition that there exists a continuous analytic selector for $\left\{\gamma_{z}\right\}_{z \in \partial \Sigma}$. The following theorem shows that the existence of solutions of the Riemann-Hilbert problem for $\left\{\gamma_{z}\right\}_{z \in \partial \Sigma}$ is actually equivalent to the existence of a 'bounded' analytic selector for $\left\{\gamma_{z}\right\}_{z \in \partial \Sigma}$.

Theorem 1.4. Let $\Sigma$ be a bordered Riemann surface with genus $g$ and $m$ real analytic boundary components. Let $\left\{\gamma_{z}\right\}_{z \in \partial \Sigma}$ be a $C^{k+1}(k \geq 3)$ family of Jordan curves in $\mathbb{C}$. Either there is no bounded holomorphic function $f$ on $\Sigma$ such that $f(z) \in \widehat{\gamma_{z}}$ almost everywhere on $\partial \Sigma$, or there exists a solution of the RiemannHilbert problem for $\left\{\gamma_{z}\right\}_{z \in \partial \Sigma}$. In the latter case there always exists a solution $f_{0}$ of the Riemann-Hilbert problem for $\left\{\gamma_{z}\right\}_{z \in \partial \Sigma}$ such that the winding number of the outer normal to $\gamma_{z}$ at $f_{0}(z)$ along $\partial \Sigma$ is at most $2 g+m-1$.

Let $A(\Sigma \times \mathbb{C})$ denote the space of all continuous functions on $\bar{\Sigma} \times \mathbb{C}$, which are holomorphic on $\Sigma \times \mathbb{C}$, equipped with the topology of the uniform convergence on compact subsets of $\bar{\Sigma} \times \mathbb{C}$. Recall that the $A(\Sigma \times \mathbb{C})$-hull $\widehat{K}$ of a compact set $K \subseteq \bar{\Sigma} \times \mathbb{C}$ is defined as

$$
\widehat{K}=\left\{(z, w) \in \bar{\Sigma} \times \mathbb{C} ;|h(z, w)| \leq \max _{K}|h| \text { for every } h \in A(\Sigma \times \mathbb{C})\right\} .
$$

Let

$$
T=\bigcup_{z \in \partial \Sigma}\left(\{z\} \times \gamma_{z}\right) .
$$

Then $T$ is the union of $m$ totally real tori in $\partial \Sigma \times \mathbb{C}$, and by the maximum principle the graph of every function from the family $\mathcal{F}$ belongs to the $A(\Sigma \times \mathbb{C})$-hull of $T$. In the case of the disc $\Sigma=\Delta$ it follows from results in [3], 20] and [29] that the (polynomial) $A(\Delta \times \mathbb{C}$ )-hull of $T$ over $\Sigma$ equals the union of graphs of functions from $\mathcal{F}$. For a bordered Riemann surface $\Sigma \neq \Delta$ it is known that in general $\widehat{T} \cap(\Sigma \times \mathbb{C})$ cannot be given as the union of graphs of functions from $\mathcal{F}$ (it might even happen that $\widehat{T} \cap(\Sigma \times \mathbb{C}) \neq \emptyset$ but $\mathcal{F}=\emptyset$, [4, 16]), however Theorem 1.1] gives some 'lower' bound on the size and geometry of the $A(\Sigma \times \mathbb{C})$-hull of $T$.

\section{Extremal FunCtions}

The standard normal family argument and the next lemma show that there exists a function $f_{0} \in \mathcal{F}$ such that

$$
f_{0}(p)=\max \{f(p) ; f \in \mathcal{F}\} .
$$

Lemma 2.1. For every $z_{0} \in \partial \Sigma$ we have

$$
\widehat{\gamma_{z_{0}}}=\widehat{T} \cap\left(\left\{z_{0}\right\} \times \mathbb{C}\right) .
$$

Proof. Let $w_{0} \notin \widehat{\gamma_{z_{0}}}$. Since $\widehat{\gamma_{z_{0}}}$ is simply connected, there exists a polynomial $Q(w)$ so that $\|Q\|_{\widehat{\gamma_{0}}}<Q\left(w_{0}\right)=1$. Let $\varphi$ be an Ahlfors function on $\Sigma$ which takes $z_{0}$ to 1 and let $\varphi^{-1}(1)=\left\{z_{0}, z_{1}, \ldots, z_{r}\right\}$. Let $\mu$ be a holomorphic function on $\Sigma$ smooth 
up to the boundary such that $\mu\left(z_{0}\right)=1$ and $\mu\left(z_{1}\right)=\cdots=\mu\left(z_{r}\right)=0$. We consider functions

$$
(z, w) \longmapsto F_{n}(z, w)=\mu(z) \frac{1}{(2-\varphi(z))^{n}} Q(w)
$$

from $A(\Sigma \times \mathbb{C})$. Then $F_{n}\left(z_{0}, w_{0}\right)=1$ for every $n \in \mathbb{N}$. By continuity it follows that there is a neighbourhood $U$ of $z_{0}$ on $\partial \Sigma$ so that for every $n \in \mathbb{N}$ and every $z \in U$ it holds that

$$
\left\|\mu(z) \frac{1}{(2-\varphi(z))^{n}} Q\right\|_{\widehat{\gamma_{z}}}<1 .
$$

On the other hand we have that the sequence $\left\{\mu(z) \frac{1}{(2-\varphi(z))^{n}}\right\}_{n \in \mathbb{N}}$ converges uniformly on compact subsets of $\bar{\Sigma} \backslash\left\{z_{0}\right\}$ to 0 . The boundedness of $Q$ on $T$ implies that there exists $n \in \mathbb{N}$ so that $\left\|F_{n}\right\|_{T}<1$. Hence $\left(z_{0}, w_{0}\right)$ is not in $\widehat{T}$ and so $\widehat{T} \cap\left(\left\{z_{0}\right\} \times \mathbb{C}\right) \subseteq \widehat{\gamma_{z_{0}}}$. The reverse inclusion is obvious.

To proceed with the proof of Theorem 1.1 we define different families of holomorphic functions on $\Sigma$ we will need in our argument. We will denote by $\mathcal{F}_{C}$ the family of all continuous up to the boundary holomorphic functions from $\mathcal{F}$, and we will denote by $\mathcal{F}_{R H}$ the family of all functions from $\mathcal{F}_{C}$ which solve the RiemannHilbert problem for $\left\{\gamma_{z}\right\}_{z \in \partial \Sigma}$. It is a consequence of Cirka's theorem [15] on the regularity of an analytic set with boundary in a maximal real manifold that each member of $\mathcal{F}_{R H}$ is of class $C^{k, \alpha}$. On the other hand it follows from [12] that $\mathcal{F}_{R H}$ is nonempty. Namely, let $g \in A^{k+1}(\Sigma)$ be a holomorphic function such that it has the only simple zero on $\bar{\Sigma}$ at point $p$. We consider the Riemann-Hilbert problem for the $C^{k+1}$ family of Jordan curves in $\mathbb{C}$ defined as

$$
\widetilde{\gamma}_{z}=\frac{1}{g(z)} \gamma_{z}
$$

This family still has the property that each curve $\widetilde{\gamma}_{z}(z \in \partial \Sigma)$ contains the point 0 in their interior. Let $h$ be a solution of the corresponding Riemann-Hilbert problem. Then $f=g h \in \mathcal{F}_{R H}$. Finally, for each $n \in \mathbb{N} \cup\{0\}$ we define the family $\mathcal{F}_{n}$ of all functions from $\mathcal{F}_{R H}$ which have at most $n$ zeros on $\Sigma$. Then $\mathcal{F}_{0} \subseteq \mathcal{F}_{1} \subseteq \cdots \subseteq$ $\mathcal{F}_{R H} \subseteq \mathcal{F}_{C}$, and there exists $n_{0} \in \mathbb{N} \cup\{0\}$ such that $\mathcal{F}_{n} \neq \emptyset$ for all $n \geq n_{0}$.

It follows from results in [12, 21], 26] and [32] that each family $\mathcal{F}_{n}(n \in \mathbb{N} \cup\{0\})$ is compact in Gromov's topology, that is, if $\left\{f_{l}\right\} \subseteq \mathcal{F}_{n}$ is a sequence, then there exists a subsequence $\left\{f_{l_{j}}\right\}$, a finite set $\Gamma \subset \partial \Sigma$ and a holomorphic function $f_{\infty} \in \mathcal{F}_{n}$ such that $\left\{f_{l_{j}}\right\}$ converges to $f_{\infty}$ in the $C^{k, \alpha}$ sense on compact subsets of $\bar{\Sigma} \backslash \Gamma$.

The compactness tells us that for each $n \geq n_{0}$ there exists a function $f_{n} \in \mathcal{F}_{n}$ so that

$$
f_{n}(p)=\max \left\{f(p) ; f \in \mathcal{F}_{n}\right\} .
$$

The following lemma implies that all these functions belong to $\mathcal{F}_{2 g+m-1}$.

Lemma 2.2. Let $f \in \mathcal{F}_{R H}$ be a solution of the Riemann-Hilbert problem for $\left\{\gamma_{z}\right\}_{z \in \partial \Sigma}$ with more than $2 g+m-1$ zeros on $\Sigma$. Then there exists $\widetilde{f}$, a solution of the Riemann-Hilbert problem for $\left\{\gamma_{z}\right\}_{z \in \partial \Sigma}$ so that

$$
\widetilde{f}(p)>f(p) .
$$

Proof. Let $f \in A^{k, \alpha}(\Sigma) \cap \mathcal{F}$ be a solution of the Riemann-Hilbert problem for $\left\{\gamma_{z}\right\}_{z \in \partial \Sigma}$ with more than $2 g+m-1$ zeros. We will show that it is possible to 
slightly perturb $f$ and get another solution $\tilde{f}$ of the Riemann-Hilbert problem so that $\widetilde{f}(p)>f(p)$.

Let $\rho$ be a $C^{k+1}$ defining function for $\left\{\gamma_{z}\right\}_{z \in \partial \Sigma}$. The map

$$
\begin{gathered}
\Psi: A^{1, \alpha}(\Sigma) \longrightarrow C^{1, \alpha}(\partial \Sigma), \\
(\Psi(h))(z)=\rho(z, h(z))
\end{gathered}
$$

is $C^{1}$ [22], and its derivative at $f \in A^{1, \alpha}(\Sigma)$ is

$$
(D \Psi(f) h)(z)=2 \operatorname{Re}\left(\left(\partial_{w} \rho\right)(z, f(z)) h(z)\right) .
$$

Geometric assumptions on the family of simple closed curves $\left\{\gamma_{z}\right\}_{z \in \partial \Sigma}$ imply that the winding number of the normal to $\gamma_{z}$ at $f(z)$, that is, the winding number of the nonzero function $a(z)=\left(\bar{\partial}_{w} \rho\right)(z, f(z))$ on $\partial \Sigma$, equals the winding number of $f$ on $\partial \Sigma$ which in turn is equal to the number $n$ of zeros of $f$.

The linear operator $D \Psi(f)$ is a Fredholm operator of index $2 n-(2 g+m-2)$, and it has no cokernel if $n \geq 2 g+m-1$, 24]. The implicit function theorem shows that in the case $n \geq 2 g+m-1$ the family $\mathcal{M}$ of all nearby to $f$ solutions of the Riemann-Hilbert problem for $\left\{\gamma_{z}\right\}_{z \in \partial \Sigma}$ is a $q=2 n-(2 g+m-2)$ dimensional submanifold of $A^{1, \alpha}(\Sigma)$.

Let $f(\cdot, s)$ be a local $C^{1}$ parametrization of $\mathcal{M}$ with the parameter space $0 \in$ $\mathcal{S} \subseteq \mathbb{R}^{q}$ such that $f(\cdot, 0)=f$. The derivative $\left(D_{s} f\right)(\cdot, 0)$ is an isomorphism from $\mathbb{R}^{q}$ onto the tangent space of $\mathcal{M}$ at $f$, that is, for every $s \in \mathbb{R}^{q}$ we have

$$
\operatorname{Re}\left(\overline{a(z)}\left(D_{s} f\right)(z, 0) s\right)=0 \quad(z \in \partial \Sigma) .
$$

We consider the mapping $\Phi: \mathcal{S} \rightarrow \mathbb{C}$ defined by $\Phi(s)=f(p, s)$. We will show that $\Phi$ is a submersion in a neighbourhood of $0 \in \mathcal{S}$. Therefore for every value $v$ close enough to $f(p)$ the equation $\Phi(s)=v$ has a solution $s(v)$ and $\widetilde{f}=f(\cdot, s(v)) \in$ $A^{1, \alpha}(\Sigma)$ is found. By a theorem of Črka, [15], we also have $\tilde{f} \in A^{k, \alpha}(\Sigma)$.

To prove that the derivative $(D \Phi)(0): \mathbb{R}^{q} \rightarrow \mathbb{C}$ is surjective we have to prove that the partial derivative $\left(D_{s} f\right)(p, 0): \mathbb{R}^{q} \rightarrow \mathbb{C}$ is surjective.

We argue by contradiction. Let us assume that the image of $\left(D_{s} f\right)(p, 0)$ is either 0 or 1 dimensional. In either case its image lies in a line in $\mathbb{R}^{2}$, and we may assume, without loss of generality, that its image is contained in the real line, that is,

$$
\operatorname{Re}\left(i\left(D_{s} f\right)(p, 0) s\right)=0
$$

for every $s \in \mathbb{R}^{q}$.

Let $h_{j}(z)=\left(D_{s} f\right)(z, 0) e_{j}, j=1, \ldots, q$, be the image of the standard basis of the space $\mathbb{R}^{q}$. Then $h_{j}(p) \in \mathbb{R}$ for each $j$ and hence there is another basis $\widetilde{e}_{j}$, $j=1, \ldots, q$, of $\mathbb{R}^{q}$ so that for their images $\widetilde{h}_{j}(z)=\left(D_{s} f\right)(z, 0) \widetilde{e}_{j}$ we have $\widetilde{h}_{j}(p)=0$, $j=1, \ldots, q-1$.

From (2.1) it follows that

$$
\operatorname{Re}\left(\overline{a(z)} \widetilde{h}_{j}(z)\right)=0
$$

for every $z \in \partial \Sigma$ and $j=1, \ldots, q$. Let $g \in A^{k, \alpha}(\Sigma)$ be such that it has the only simple zero on $\bar{\Sigma}$ at point $p$. Then $\widetilde{h}_{j}(z)=g(z) g_{j}(z)$ for some $g_{j} \in A^{k, \alpha}(\Sigma)$, $j=1, \ldots, q-1$. Therefore

$$
\operatorname{Re}\left(\overline{a(z)} g(z) g_{j}(z)\right)=0
$$

for every $z \in \partial \Sigma$ and $j=1, \ldots, q-1$, and the linear independence of functions $\widetilde{h}_{j}$, $j=1, \ldots, q-1$, implies the linear independence of functions $g_{j}, j=1, \ldots, q-1$. 
For $z \in \partial \Sigma$ we define

$$
b(z)=a(z) \overline{g(z)} .
$$

Then $b$ is a nonzero function on $\partial \Sigma$ of class $C^{k, \alpha}$, and its winding number on $\partial \Sigma$ is

$$
W(b)=W(a)-W(g)=W(a)-1=n-1 .
$$

Hence $W(b) \geq 2 g+m-1$, and the space of solutions of the linear homogeneous Riemann-Hilbert problem $\operatorname{Re}(\overline{b(z)} h(z))=0$ is $2(n-1)-(2 g+m-2)=q-2$ dimensional, 24. Thus the functions $g_{j}, j=1, \ldots, q-1$, have to be linearly dependent, which is a contradiction.

Let us summarize our results.

\section{Lemma 2.3.}

$$
\max \left\{f(p) ; f \in \mathcal{F}_{R H}\right\}=\max \left\{f(p) ; f \in \mathcal{F}_{2 g+m-1}\right\} .
$$

Proof. Lemma 2.2 and Gromov's compactness imply

$$
\max \left\{f(p) ; f \in \mathcal{F}_{2 g+m-1}\right\}=\max \left\{f(p) ; f \in \mathcal{F}_{n}\right\}
$$

for every $n \geq 2 g+m-1$.

Let $\varepsilon>0$ and let $f_{\varepsilon} \in \mathcal{F}_{R H}$ be such that

$$
f_{\varepsilon}(p)>\sup \left\{f(p) ; f \in \mathcal{F}_{R H}\right\}-\varepsilon .
$$

Function $f_{\varepsilon}$ has finitely many zeros on $\Sigma$, and hence there is $n \geq 2 g+m-1$ so that $f_{\varepsilon} \in \mathcal{F}_{n}$. Therefore

$$
\max \left\{f(p) ; f \in \mathcal{F}_{2 g+m-1}\right\} \geq f_{\varepsilon}(p) \geq \sup \left\{f(p) ; f \in \mathcal{F}_{R H}\right\}-\varepsilon .
$$

Since $\varepsilon>0$ was arbitrary, we get

$$
\max \left\{f(p) ; f \in \mathcal{F}_{2 g+m-1}\right\} \geq \sup \left\{f(p) ; f \in \mathcal{F}_{R H}\right\} .
$$

The reverse inequality is obvious.

Recall ([12]) that there exists a $C^{k+1}$ strongly plurisubharmonic function $v$ on $\bar{\Sigma} \times \mathbb{C}$ such that $T=\bigcup_{z \in \partial \Sigma}\left(\{z\} \times \gamma_{z}\right)$ is a Lagrangian submanifold for the symplectic form $\omega=i \partial \bar{\partial} v$ and that the $\omega$-area of any fiber $\{z\} \times \widehat{\gamma_{z}}$ is 1 .

Let $X=\left(0, X_{0}\right)$ be a $C^{k+1}$ vertical vector field on $\partial \Sigma \times \mathbb{C}$ with the following properties $([29])$ :

1. $X_{0}(z, w)$ is transversal to $\gamma_{z}$ for every $z \in \partial \Sigma$ and $w \in \gamma_{z}$.

2. There are $0<r<R<\infty$ so that $X_{0}(z, w)=w$ for every $z \in \partial \Sigma$ and $w \in \mathbb{C}$ such that $|w| \leq r$ or $|w| \geq R$.

3. $X_{0}(z, w)=0$ if and only if $w=0$.

Let $\Phi^{t}$ be the flow of vector field $X$ and let

$$
\{z\} \times \gamma_{z}^{t}=\Phi^{t}\left(\{z\} \times \gamma_{z}\right) .
$$

Let $A(z, t)=\int_{\{z\} \times \widehat{\gamma_{z}^{t}}} \omega$ be the $\omega$-area of the fiber $\{z\} \times \widehat{\gamma_{z}^{t}}$. Then $A(z, t) \in$ $C^{k+1}(\partial \Sigma \times \mathbb{R})$ and the properties of vector field $X$ and the fact that $v$ is a strongly plurisubharmonic function imply that $A(z, t)$ has a nonvanishing $t$-derivative.

Let $a>0$. For each $z \in \partial \Sigma$ there exists exactly one time $t(z, a)$ such that $A(z, t(z, a))=a$, and by the implicit function theorem this dependence is $C^{k+1}$ 
smooth in $(z, a) \in \partial \Sigma \times(0, \infty)$. Let $\pi_{w}: \bar{\Sigma} \times \mathbb{C} \rightarrow \mathbb{C}$ be the projection on the second coordinate. The family

$$
\left\{\pi_{w}\left(\Phi^{t(z, a)}\left(\{z\} \times \gamma_{z}\right)\right)\right\}_{z \in \partial \Sigma}
$$

of $C^{k+1}$ Jordan curves in $\mathbb{C}$ has the property that the $\omega$-area of any fiber is $a$.

The next lemma takes care of functions from $\mathcal{F}$ continuous up to the boundary.

Lemma 2.4.

$$
\max \left\{f(p) ; f \in \mathcal{F}_{R H}\right\}=\max \left\{f(p) ; f \in \mathcal{F}_{C}\right\} .
$$

Proof. Let $\varepsilon>0$ and let $f_{\varepsilon} \in \mathcal{F}_{C}$ be such that

$$
f_{\varepsilon}(p)>\sup \left\{f(p) ; f \in \mathcal{F}_{C}\right\}-\varepsilon .
$$

There exists $\left\{\gamma_{z}^{n}\right\}_{z \in \partial \Sigma}, n \in \mathbb{N}$, a sequence of $C^{k+1}$ families of simple closed curves in $\mathbb{C}$ such that the $\omega$-area of any fiber $\{z\} \times \widehat{\gamma_{z}^{n}}$ is a constant which depends only on $n$,

for every $n \in \mathbb{N}$, and

$$
\widehat{\gamma_{z}^{n+1}} \subseteq \operatorname{Int}\left(\gamma_{z}^{n}\right) \quad(z \in \partial \Sigma)
$$

$$
\bigcap_{n \in \mathbb{N}} \widehat{\gamma_{z}^{n}}=\widehat{\gamma_{z}} \quad(z \in \partial \Sigma) .
$$

It follows from results in 12 that for each $n \in \mathbb{N}$ there exists $g_{n} \in A^{k, \alpha}(\Sigma)$ a solution of the Riemann-Hilbert problem for $\left\{\gamma_{z}^{n}\right\}_{z \in \partial \Sigma}$ such that $f_{\varepsilon}(p) \leq g_{n}(p)$. To get $g_{n}$ let $\delta_{n}>0$ be so small that the disc $\Delta\left(f_{\varepsilon}(z), 3 \delta_{n}\right)$ is contained in $\widehat{\gamma_{z}^{n}}$ for every $z \in \partial \Sigma$. Then there exists $([10,[23)$ a smooth up to the boundary holomorphic function $f_{\varepsilon}^{n}$ such that $\left\|f_{\varepsilon}^{n}-f_{\varepsilon}\right\|_{\infty}<\delta_{n}$ on $\bar{\Sigma}$. Also, let $g \in A^{k+1}(\Sigma)$ be such that it has the only simple zero on $\bar{\Sigma}$ at point $p$. We consider the Riemann-Hilbert problem for the family of $C^{k+1}$ Jordan curves

$$
\widetilde{\gamma}_{z}^{n}=\frac{1}{g(z)}\left(\gamma_{z}^{n}-f_{\varepsilon}^{n}(z)-\left(f_{\varepsilon}(p)-f_{\varepsilon}^{n}(p)\right)-\delta_{n}\right)
$$

which all contain 0 in their interior. Let $h_{n}$ be a solution of the corresponding Riemann-Hilbert problem. Then

$$
g_{n}=f_{\varepsilon}^{n}+\left(f_{\varepsilon}(p)-f_{\varepsilon}^{n}(p)\right)+\delta_{n}+g h_{n} .
$$

Lemma 2.3 implies that there exists a sequence of holomorphic functions $\left\{f_{n}\right\}_{n \in \mathbb{N}}$ on $\Sigma$ of class $C^{k, \alpha}$ such that

a) $f_{n}$ solves the Riemann-Hilbert problem for $\left\{\gamma_{z}^{n}\right\}_{z \in \partial \Sigma}$.

b) $f_{n}$ has at most $2 g+m-1$ zeros on $\Sigma$.

c) $f_{n}(p) \geq f_{\varepsilon}(p) \geq \sup \left\{f(p) ; f \in \mathcal{F}_{C}\right\}-\varepsilon$.

By Gromov's compactness theorem [21, 26], 32] there exists a subsequence $\left\{f_{n_{j}}\right\}$, a finite set $\Gamma \subset \partial \Sigma$ and a holomorphic function $f_{\infty} \in \mathcal{F}_{R H}$ such that $\left\{f_{n_{j}}\right\}$ converges in the $C^{k, \alpha}$ sense on compact subsets of $\bar{\Sigma} \backslash \Gamma$ to $f_{\infty}$, a solution of the RiemannHilbert problem for $\left\{\gamma_{z}\right\}_{z \in \partial \Sigma}$. Hence

$$
\max \left\{f(p) ; f \in \mathcal{F}_{R H}\right\} \geq f_{\infty}(p) \geq \sup \left\{f(p) ; f \in \mathcal{F}_{C}\right\}-\varepsilon .
$$

Since $\varepsilon>0$ was arbitrary we get

$$
\max \left\{f(p) ; f \in \mathcal{F}_{R H}\right\} \geq \sup \left\{f(p) ; f \in \mathcal{F}_{C}\right\} .
$$

The reverse inequality is trivial. 
The following lemma completes the proof of Theorem 1.1

\section{Lemma 2.5.}

$$
\max \{f(p) ; f \in \mathcal{F}\}=\max \left\{f(p) ; f \in \mathcal{F}_{C}\right\}
$$

Proof. Let $\left\{\gamma_{z}^{n}\right\}_{z \in \partial \Sigma}, n \in \mathbb{N}$, be a sequence of $C^{k+1}$ families of Jordan curves in $\mathbb{C}$ as in the proof of Lemma 2.4 such that the $\omega$-area of any fiber $\{z\} \times \widehat{\gamma_{z}^{n}}$ is a constant which depends only on $n$,

$$
\widehat{\gamma_{z}^{n+1}} \subseteq \operatorname{Int}\left(\gamma_{z}^{n}\right) \quad(z \in \partial \Sigma)
$$

for every $n \in \mathbb{N}$, and

$$
\bigcap_{n \in \mathbb{N}} \widehat{\gamma_{z}^{n}}=\widehat{\gamma_{z}} \quad(z \in \partial \Sigma) .
$$

Let $\left\{\Sigma_{l}\right\}_{l \in \mathbb{N}}$ be an increasing sequence $p \in \overline{\Sigma_{l}} \subseteq \Sigma_{l+1}(l \in \mathbb{N})$ of domains in $\Sigma$ with real analytic boundaries, of the same topological type as $\Sigma$, and such that their union is $\Sigma$. Let $J$ be the complex structure on $\Sigma$. Then $\left(\Sigma_{l}, J\right)$ is a sequence of Riemann surfaces which 'converges' to $(\Sigma, J)$, that is, let $\left\{\psi_{l}\right\}_{l \in \mathbb{N}}$ be a sequence of smooth diffeomorphisms $\psi_{l}: \bar{\Sigma} \rightarrow \overline{\Sigma_{l}}, \psi_{l}(p)=p$, which in the $C^{\infty}$ sense converges to the identity map, and let

$$
J_{l}=\left(D \psi_{l}\right)^{-1} \circ J \circ D \psi_{l} .
$$

Then $\left\{J_{l}\right\}_{l \in \mathbb{N}}$ is a sequence of complex structures on $\bar{\Sigma}$ which $C^{\infty}$ converges to $J$.

Let $n \in \mathbb{N}$ be fixed and let $f_{0} \in \mathcal{F}$ be such that

$$
f_{0}(p)=\max \{f(p) ; f \in \mathcal{F}\} .
$$

For every $l$ we define $f_{l}=f_{0} \circ \psi_{l}$ a smooth up to the boundary holomorphic function on $\left(\Sigma, J_{l}\right)$.

Since the graph of $f_{0}$ belongs to a $A(\Sigma \times \mathbb{C})$-hull of the tori

$$
T=\bigcup_{z \in \partial \Sigma}\left(\{z\} \times \gamma_{z}\right),
$$

which forms a closed subset of $\bar{\Sigma} \times \mathbb{C}$, we get that there exists $l_{0} \in \mathbb{N}$ so that for every $l>l_{0}$ we have

$$
f_{l}(z) \in \operatorname{Int}\left(\gamma_{z}^{n}\right) \quad(z \in \partial \Sigma) .
$$

If not, there exists a sequence $l_{j} \rightarrow \infty$ and a sequence $z_{j} \in \partial \Sigma$ which converges to $z_{0} \in \partial \Sigma$ such that

$$
w_{j}=f_{l_{j}}\left(z_{j}\right)=f_{0}\left(\psi_{l_{j}}\left(z_{j}\right)\right) \notin \operatorname{Int}\left(\gamma_{z_{j}}^{n}\right) .
$$

Since $f_{0}$ is bounded, there exists, after passing to a subsequence, the limit $w_{0}$ of sequence $\left\{w_{j}\right\}_{j \in \mathbb{N}}$. Therefore the sequence

$$
\left(\psi_{l_{j}}\left(z_{j}\right), w_{j}\right)=\left(\psi_{l_{j}}\left(z_{j}\right), f_{0}\left(\psi_{l_{j}}\left(z_{j}\right)\right)\right)
$$

of points from the graph of $f_{0}$ converges to $\left(z_{0}, w_{0}\right) \notin\left\{z_{0}\right\} \times \widehat{\gamma_{z_{0}}}$, which is a contradiction.

By Lemma 2.4 there exists a sequence $\left\{g_{l}\right\}_{l>l_{0}}$ of $C^{k, \alpha}$ functions on $\bar{\Sigma}$ such that for every $l>l_{0}$

a) $g_{l}$ is holomorphic on $\left(\Sigma, J_{l}\right)$.

b) $g_{l}$ solves the Riemann-Hilbert problem on $\left(\Sigma, J_{l}\right)$ for $\left\{\gamma_{z}^{n}\right\}_{z \in \partial \Sigma}$.

c) $g_{l}$ has at most $2 g+m-1$ zeros on $\Sigma$.

d) $g_{l}(p) \geq f_{l}(p)=f_{0}\left(\psi_{l}(p)\right)=f_{0}(p)$. 
Let $l \rightarrow \infty$. Using Gromov's compactness theorem we get that there exists a $C^{k, \alpha}(\Sigma)$ holomorphic function $\widetilde{f}_{n}$ on $(\Sigma, J)$ such that

a) $\tilde{f}_{n}$ solves the Riemann-Hilbert problem on $(\Sigma, J)$ for $\left\{\gamma_{z}^{n}\right\}_{z \in \partial \Sigma}$.

b) $\widetilde{f}_{n}$ has at most $2 g+m-1$ zeros on $\Sigma$.

c) $\widetilde{f}_{n}(p) \geq f_{0}(p)=\max \{f(p) ; f \in \mathcal{F}\}$.

Let $n \rightarrow \infty$, and the proof is finished as in Lemma 2.4.

A similar argument also gives the following theorem.

Theorem 2.6. Let $\Sigma$ be a bordered Riemann surface with genus $g$ and $m$ real analytic boundary components. Let $\left\{\gamma_{z}\right\}_{z \in \partial \Sigma}$ be a $C^{k+1}(k \geq 3)$ family of Jordan curves in $\mathbb{C}$ which all contain the point 0 in their interior. Then there exists a holomorphic function $f_{0} \in A^{k, \alpha}(\Sigma)$ with at most $2 g+m-1$ zeros on $\Sigma$ so that $f_{0}(z) \in \gamma_{z}$ for every $z \in \partial \Sigma$ and such that

$$
\left.\left|f_{0}(p)\right|=\max \left\{|f(p)| ; f \in H^{\infty}(\Sigma), f(z) \in \widehat{\gamma_{z}} \text { (a.e. } z \in \partial \Sigma\right)\right\} .
$$

Proof of Corollary 1.3. Here we prove the existence part of Corollary 1.3. The uniqueness part is proved in the next section.

Let $g$ be a smooth up to the boundary holomorphic function on $\Sigma$ such that $(g)=\mathcal{D}$ and $g^{(n)}(p)>0$ in a given coordinate chart. Let us consider the $C^{k+1}$ family of Jordan curves in $\mathbb{C}$ defined as

$$
\widetilde{\gamma}_{z}=\frac{1}{g(z)} \gamma_{z}
$$

By Theorem 1.1 there exists a solution of the corresponding Riemann-Hilbert problem $f_{1} \in \mathcal{F} \cap A^{k, \alpha}(\Sigma)$ with at most $2 g+m-1$ zeros on $\Sigma$ so that $f_{1}(p) \geq f(p)$ for every bounded holomorphic function $f$ on $\Sigma$, such that $f(p) \geq 0$ and $f(z) \in \widehat{\widetilde{\gamma}}_{z}$ for almost every $z \in \partial \Sigma$. Finally we define $f_{0}=g f_{1}$.

\section{UNIQUENESS}

In this section we show the uniqueness of the extremal functions in the case where the given smooth family of smooth Jordan curves in $\mathbb{C}$ consists of convex curves which are strictly convex on a set $z \in \partial \Sigma$ with positive measure. This follows immediately once we prove that in this case every extremal function $f$ is 'almost proper', that is, $f(z) \in \gamma_{z}$ for almost every $z \in \partial \Sigma$. Namely, let $f_{1}$ and $f_{2}$ be two different extremal function for $p \in \Sigma$. Then $f_{1}(z) \neq f_{2}(z)$ for almost every $z \in \partial \Sigma$, and since $\left\{\gamma_{z}\right\}_{z \in \partial \Sigma}$ are strictly convex on a set $z \in \partial \Sigma$ with positive measure, we get that $f=\left(f_{1}+f_{2}\right) / 2$ is an extremal function such that $f(z) \in \operatorname{Int}\left(\gamma_{z}\right)$ on a set $z \in \partial \Sigma$ with positive measure. This leads to a contradiction, and we get uniqueness.

Let $K_{1}, \ldots, K_{2 g+m-1}$ be oriented smooth simple closed curves in $\Sigma$ which form a canonical basis for $H_{1}(\Sigma)$, 24, 28. Let $u$ be a harmonic function on $\Sigma$. For each canonical cycle $K_{j}, j=1, \ldots, 2 g+m-1$, we assign $u$ its period along $K_{j}$,

$$
P_{j}(u)=\frac{1}{\pi i} \int_{K_{j}} \partial u
$$

Here, $\partial u=\frac{1}{2}(d u-i d u \circ J)$, where $J$ is the complex structure on $\Sigma$. If $u$ is a real harmonic function on $\Sigma$, all its periods are real. 
Remark 3.1. The periods of a harmonic function $u$ can also be defined using operator $d^{c}=i(\bar{\partial}-\partial)=-d u \circ J$,

$$
P_{j}(u)=\frac{1}{2 \pi} \int_{K_{j}} d^{c} u
$$

Recall that a harmonic function $u$ on $\Sigma$ has a well-defined harmonic conjugate if and only if all its periods are 0 . On the other hand, if this condition is not satisfied, one can still define, via the integration, a multiple-valued harmonic conjugate of $u$. In a special case when all the periods of a real harmonic function $u$ are integers, the harmonic conjugate of $u$ might not be well defined, however its composition with the exponential function is a well-defined function on $\Sigma$, and we get a holomorphic function $f$ on $\Sigma$ such that

$$
|f(z)|=e^{u(z)} \quad(z \in \Sigma) .
$$

We claim that there exist $2 g+m-1$ smooth up to the boundary real harmonic functions $h_{1}, \ldots, h_{2 g+m-1}$ on $\Sigma$ such that the matrix

$$
P=\left[\begin{array}{ccc}
P_{1}\left(h_{1}\right) & \ldots & P_{1}\left(h_{2 g+m-1}\right) \\
\vdots & \ddots & \vdots \\
P_{2 g+m-1}\left(h_{1}\right) & \ldots & P_{2 g+m-1}\left(h_{2 g+m-1}\right)
\end{array}\right]
$$

is invertible.

Let $G: \bar{\Sigma} \times \bar{\Sigma} \rightarrow[-\infty, 0]$ be the Green's function on $\Sigma$. For every real smooth function $\varphi$ on $\partial \Sigma$ we define

$$
\Phi(\varphi)(z)=\frac{2}{i} \int_{\partial \Sigma} \partial_{w} G(z, w) \varphi(w)
$$

Then $\Phi(\varphi)$ is a smooth up to the boundary real harmonic function on $\Sigma$ such that $\left.\Phi(\varphi)\right|_{\partial \Sigma}=\varphi$.

Let us observe the linear map which to any real harmonic function $u$ on $\Sigma$ assigns all its periods

$$
u \longmapsto\left(P_{1}(u), \ldots, P_{2 g+m-1}(u)\right) \in \mathbb{R}^{2 g+m-1} .
$$

If there are no smooth up to the boundary real harmonic functions $h_{1}, \ldots, h_{2 g+m-1}$ on $\Sigma$ such that matrix $P$ is invertible, then there exist real numbers $\lambda_{1}, \ldots, \lambda_{2 g+m-1}$, which are not all equal to 0 , such that

$$
\lambda_{1} P_{1}(u)+\cdots+\lambda_{2 g+m-1} P_{2 g+m-1}(u)=0
$$

for every real harmonic function $u$ on $\Sigma$. Thus for every real smooth function $\varphi$ on $\Sigma$ we have

$$
\lambda_{1} P_{1}(\Phi(\varphi))+\cdots+\lambda_{2 g+m-1} P_{2 g+m-1}(\Phi(\varphi))=0 .
$$

Let us compute

$$
P_{j}(\Phi(\varphi))=-\frac{2}{\pi} \int_{K_{j}} \partial_{z}\left(\int_{\partial \Sigma} \partial_{w} G(z, w) \varphi(w)\right)=-\frac{2}{\pi} \int_{\partial \Sigma} \varphi(w) \partial_{w}\left(\int_{K_{j}} \partial_{z} G(z, w)\right) .
$$

Therefore for every real smooth function $\varphi$ on $\partial \Sigma$ we have

$$
\int_{\partial \Sigma} \varphi(w)\left(\lambda_{1} \partial_{w}\left(\int_{K_{1}} \partial_{z} G(z, w)\right)+\cdots+\lambda_{2 g+m-1} \partial_{w}\left(\int_{K_{2 g+m-1}} \partial_{z} G(z, w)\right)\right)=0
$$

and hence

$$
\lambda_{1} \partial_{w}\left(\int_{K_{1}} \partial_{z} G(z, w)\right)+\cdots+\lambda_{2 g+m-1} \partial_{w}\left(\int_{K_{2 g+m-1}} \partial_{z} G(z, w)\right)=0
$$


for every $w \in \partial \Sigma$. It is known $([28])$ that $Z_{j}(w)=\partial_{w}\left(\int_{K_{j}} \partial_{z} G(z, w)\right), j=$ $1, \ldots, 2 g+m-1$, are $\mathbb{R}$ linearly independent holomorphic differentials on $\Sigma$. Thus $\lambda_{1}=\cdots=\lambda_{2 g+m-1}=0$.

Lemma 3.2. Let $E \subseteq \partial \Sigma$ be a subset of positive measure. For each $\varepsilon>0$ let $\mathcal{H}_{\varepsilon}$ be the set of all bounded holomorphic functions $f$ on $\Sigma$ such that $|f| \leq 1$ almost everywhere on $E$ and $|f(z)| \leq \varepsilon$ almost everywhere on $\partial \Sigma \backslash E$. Let $p \in \Sigma$ and let $M(\varepsilon)=\sup _{\mathcal{H}_{\varepsilon}}|f(p)|$. Then

$$
\lim _{\varepsilon \downarrow 0} \frac{M(\varepsilon)}{\varepsilon}=\infty .
$$

See 27] for similar and more general results in the case of the disc.

Proof. Let $h_{1}, \ldots, h_{2 g+m-1}$ be smooth up to the boundary real harmonic functions on $\Sigma$ so that matrix $P$ of their periods (3.1) is invertible and let $K$ be the cube $[0,1]^{2 g+m-1} \subseteq \mathbb{R}^{2 g+m-1}$. Without loss of generality we may assume that $P$ is the identity matrix.

Let $\varepsilon>0$, let

$$
\chi_{\varepsilon}(z)=\left\{\begin{array}{cc}
0, & z \in E, \\
\log (\varepsilon), & z \in \partial \Sigma \backslash E,
\end{array}\right.
$$

and let $u_{\varepsilon}$ be the harmonic function on $\Sigma$ such that $u_{\varepsilon}=\chi_{\varepsilon}$ almost everywhere on $\partial \Sigma$. Let $\alpha_{j}(\varepsilon)=P_{j}\left(u_{\varepsilon}\right)-\left[P_{j}\left(u_{\varepsilon}\right)\right](j=1, \ldots, 2 g+m-1)$ and let $a(\varepsilon)=$ $\left(\alpha_{1}(\varepsilon), \ldots, \alpha_{2 g+m-1}(\varepsilon)\right) \in K$. Here, $[x]$ denotes the integer part of $x \in \mathbb{R}$.

Then all the periods of the harmonic function

$$
u_{0}=u_{\varepsilon}-\left(\alpha_{1}(\varepsilon) h_{1}+\cdots+\alpha_{2 g+m-1}(\varepsilon) h_{2 g+m-1}\right)
$$

are integers, and hence there is a well-defined holomorphic function $F$ on $\Sigma$ such that

$$
|F(z)|=e^{u_{0}(z)} \quad(z \in \Sigma) .
$$

Let

$$
C=\max \left\{\lambda_{1} h_{1}(z)+\cdots+\lambda_{2 g+m-1} h_{2 g+m-1}(z) ;\left(\lambda_{1}, \ldots, \lambda_{2 g+m-1}\right) \in K, z \in \bar{\Sigma}\right\}
$$

and let $\mu>0$ be the value at $p$ of the harmonic function on $\Sigma$ whose boundary values are almost everywhere equal to 1 on $E$ and 0 elsewhere. Then

$$
\frac{|F(p)|}{\varepsilon} \geq \varepsilon^{-\mu} e^{-C} \text {. }
$$

Also, let

$$
c=\min \left\{\lambda_{1} h_{1}(z)+\cdots+\lambda_{2 g+m-1} h_{2 g+m-1}(z) ;\left(\lambda_{1}, \ldots, \lambda_{2 g+m-1}\right) \in K, z \in \partial \Sigma\right\} .
$$

Then holomorphic function $e^{c} F(z)$ belongs to $\mathcal{H}_{\varepsilon}$ and hence

$$
\frac{M(\varepsilon)}{\varepsilon} \geq \frac{\left|e^{c} F(p)\right|}{\varepsilon} \geq \varepsilon^{-\mu} e^{c-C},
$$

which proves the lemma.

Lemma 3.3. Let $Q \subseteq \mathbb{C}$ be a compact convex set which contains the point 0 in its interior. Let $\gamma=\partial Q$ be the boundary of $Q$ and let $c=d(0, \gamma)$ be the distance of point 0 to $\gamma$. Then for every $\lambda \geq 0$ the distance of sets $\lambda \gamma$ and $\gamma$ is

$$
d(\lambda \gamma, \gamma)=c|1-\lambda| \text {. }
$$


Corollary 3.4. If $\left\{\gamma_{z}\right\}_{z \in \partial \Sigma}$ is a $C^{1}$ family of convex curves in $\mathbb{C}$ which all contain 0 in their interior, there exist constants $0<c<C<\infty$ such that

$$
c|1-\lambda| \leq d\left(\lambda \gamma_{z}, \gamma_{z}\right) \leq C|1-\lambda|
$$

for every $\lambda \geq 0$ and every $z \in \partial \Sigma$.

Proof of Lemma 3.3. We first consider the special case where $Q$ is the intersection of finitely many half-planes, that is, there exist complex numbers $a_{1}, \ldots, a_{n}$ of the unit length and positive real numbers $r_{1}, \ldots, r_{n}$ such that

$$
Q=\left\{z \in \mathbb{C}, \operatorname{Re}\left(\overline{a_{j}} z\right) \leq r_{j}, j=1, \ldots, n\right\} .
$$

A point $z \in Q$ belongs to the boundary $\gamma$ if and only if $\operatorname{Re}\left(\overline{a_{j}} z\right)=r_{j}$ for at least one $j=1, \ldots, n$.

Let $\lambda>0$, let $a$ be a unitary complex number and let $r \in \mathbb{R}$. The dilation $z \mapsto \lambda z$ maps lines to parallel lines. In particular it maps the line given by the equation $\operatorname{Re}(\bar{a} z)=r$ to the line $\operatorname{Re}(\bar{a} z)=\lambda r$, and their distance equals

$$
|1-\lambda||r| \text {. }
$$

Observe that the geometric meaning of $|r|$ is the distance of point 0 to the line $\operatorname{Re}(\bar{a} z)=r$.

The curve $\lambda \gamma$ is the boundary of the convex set

$$
\lambda Q=\left\{z \in \mathbb{C}, \operatorname{Re}\left(\overline{a_{j}} z\right) \leq \lambda r_{j}, j=1, \ldots, n\right\},
$$

and the distance $d(\lambda \gamma, \gamma)$ is the distance between two closest parallel lines which define $Q$ and $\lambda Q$. From (3.2) it follows that

$$
d(\lambda \gamma, \gamma)=c|1-\lambda|
$$

where $c=d(0, \gamma)$.

In general we know that $Q$ equals the intersection of all closed half-planes $\Pi$ such that $Q \subseteq \Pi$. Let $\lambda>0$ and let $z, w, p$ be points from $\gamma$ such that

$$
d(0, \gamma)=|p|, \quad d(\lambda \gamma, \gamma)=|\lambda w-z| .
$$

There exist complex numbers $a_{1}, \ldots, a_{n}$ of the unit length and positive real numbers $r_{1}, \ldots, r_{n}$ such that

$$
Q \subseteq \widetilde{Q}=\left\{z \in \mathbb{C}, \operatorname{Re}\left(\overline{a_{j}} z\right) \leq r_{j}, j=1, \ldots, n\right\}
$$

is a compact set which contains the points $z, w, p$ on its boundary. Let $\widetilde{\gamma}$ be the boundary of $\widetilde{Q}$. Then we have

$$
c|1-\lambda|=d(\lambda \widetilde{\gamma}, \widetilde{\gamma}) \leq d(\lambda \gamma, \gamma) \leq c|1-\lambda| .
$$

The first equality holds by the observations above and the choice of point $p$. The next inequality holds because of the choice of points $z, w$, and the last inequality is again a consequence of the choice of point $p$. This proves the lemma.

The next proposition, together with the observation from the beginning of this section, completes the proof of Theorem 1.2. See [27] for more on the 'almost properness' of the extremal functions in the disc case. 
Proposition 3.5. Let $\left\{\gamma_{z}\right\}_{z \in \partial \Sigma}$ be a $C^{1}$ family of convex Jordan curves in $\mathbb{C}$ which all contain the point 0 in their interior. Let $p \in \Sigma$ and let $\mathcal{F}$ be the family of all bounded holomorphic functions $f$ on $\Sigma$ such that $f(p) \geq 0$ and $f(z) \in \widehat{\gamma_{z}}$ for almost every $z \in \partial \Sigma$. Let $f_{0} \in \mathcal{F}$ be an extremal function, that is,

$$
f_{0}(p)=\max \{f(p) ; f \in \mathcal{F}\} .
$$

Then $f_{0}(z) \in \gamma_{z}$ for almost every $z \in \partial \Sigma$.

Proof. Let $f_{0}$ be an extremal function. Let us assume that there exists a set $E \subseteq \partial \Sigma$ of positive measure such that $f_{0}(z) \in \operatorname{Int}\left(\gamma_{z}\right)$ for every $z \in E$. Let $E_{n}=\{z \in$ $\left.E ; f_{0}(z) \in\left(1-\frac{1}{n}\right) \widehat{\gamma_{z}}\right\}$. Then $\bigcup_{n} E_{n}=E$, and hence there exists a set $E_{0} \subseteq \partial \Sigma$ of positive measure and $\lambda_{0} \in(0,1)$ such that $f_{0}(z) \in \lambda_{0} \widehat{\gamma_{z}}$ for almost every $z \in E_{0}$.

By Corollary 3.4 there exist constants $0<c<C<\infty$ such that

$$
c|1-\lambda| \leq d\left(\lambda \gamma_{z}, \gamma_{z}\right) \leq C|1-\lambda|
$$

for every $z \in \partial \Sigma$ and $\lambda \geq 0$.

Let $d_{0}=c\left(1-\lambda_{0}\right)>0$. If $E_{0}$ has full measure in $\partial \Sigma$ we are done, because we can replace $f_{0}$ with $f_{0}+d_{0} \in \mathcal{F}$. Let us now consider the case where $\partial \Sigma \backslash E_{0}$ is not of measure 0 . Let $\varepsilon>0$. The extremal value at $p$ for the family $\left\{(1+\varepsilon) \gamma_{z}\right\}_{z \in \partial \Sigma}$ is $(1+\varepsilon) f_{0}(p)$. By Lemma 3.2 there exists a family $\left\{f_{\varepsilon}\right\}_{\varepsilon>0}$ of bounded holomorphic functions on $\Sigma$ such that

1. $f_{\varepsilon}(p)>0$,

2. $\left|f_{\varepsilon}(z)\right| \leq 1$ almost everywhere on $E_{0}$,

3. $\left|f_{\varepsilon}(z)\right| \leq \frac{c}{d_{0}} \varepsilon$ almost everywhere on $\partial \Sigma \backslash E_{0}$,

and

$$
\lim _{\varepsilon \downarrow 0} \frac{f_{\varepsilon}(p)}{\varepsilon}=\infty .
$$

We define the family of functions $\left\{f_{0}+d_{0} f_{\varepsilon}\right\}_{\varepsilon>0}$ which are bounded holomorphic functions on $\Sigma$ such that $f_{0}(z)+d_{0} f_{\varepsilon}(z) \in(1+\varepsilon) \widehat{\gamma_{z}}$ almost everywhere on $\partial \Sigma$. Since the extremal value at $p$ for the family $\left\{(1+\varepsilon) \gamma_{z}\right\}_{z \in \partial \Sigma}$ is $(1+\varepsilon) f_{0}(p)$, we must have

$$
f_{0}(p)+d_{0} f_{\varepsilon}(p) \leq(1+\varepsilon) f_{0}(p)
$$

and hence

$$
\frac{f_{\varepsilon}(p)}{\varepsilon} \leq \frac{f_{0}(p)}{d_{0}}
$$

which is in contradiction with (3.3).

Corollary 3.6. Let $\left\{\gamma_{z}\right\}_{z \in \partial \Sigma}$ be a $C^{k+1}(k \geq 3)$ family of convex Jordan curves in $\mathbb{C}$ which all contain the point 0 in their interior. If there exists $z_{0} \in \partial \Sigma$ such that $\gamma_{z_{0}}$ is a strongly convex curve in $\mathbb{C}$, then there is a unique function $f_{0} \in \mathcal{F}_{R H}$ such that

$$
f_{0}(p)=\max \{f(p) ; f \in \mathcal{F}\} .
$$

Remark 3.7. A curve $\gamma \subseteq \mathbb{C}$ is strongly convex if its curvature never vanishes. 


\section{BOUNDED ANALYTIC SELECTORS}

The proof of Theorem 1.4 is similar to the proof of Lemma 2.5, and it is more or less included in it.

Let $f_{0}$ be a bounded holomorphic function on $\Sigma$ such that $f(z) \in \widehat{\gamma_{z}}$ almost everywhere on $\partial \Sigma$.

We start as in the proof of Lemma 2.5. Let $\left\{\gamma_{z}^{n}\right\}_{z \in \partial \Sigma}, n \in \mathbb{N}$, be a sequence of $C^{k+1}$ families of Jordan curves in $\mathbb{C}$ such that the $\omega$-area of any fiber $\{z\} \times \widehat{\gamma_{z}^{n}}$ is a constant which depends only on $n$,

$$
\widehat{\gamma_{z}^{n+1}} \subseteq \operatorname{Int}\left(\gamma_{z}^{n}\right) \quad(z \in \partial \Sigma)
$$

for every $n \in \mathbb{N}$, and

$$
\bigcap_{n \in \mathbb{N}} \widehat{\gamma_{z}^{n}}=\widehat{\gamma_{z}} \quad(z \in \partial \Sigma)
$$

One should observe that the condition that each curve $\gamma_{z}, z \in \partial \Sigma$, contains the point 0 in its interior is not essential for the existence of the sequence $\left\{\gamma_{z}^{n}\right\}_{z \in \partial \Sigma}$, $n \in \mathbb{N}$.

Also, let $\left\{\Sigma_{l}\right\}_{l \in \mathbb{N}}$ be an increasing sequence $\overline{\Sigma_{l}} \subseteq \Sigma_{l+1}(l \in \mathbb{N})$ of domains in $\Sigma$ with real analytic boundaries, of the same topological type as $\Sigma$, and such that their union is $\Sigma$. Let $J$ be the complex structure on $\Sigma$ and let $\left\{\psi_{l}\right\}_{l \in \mathbb{N}}$ be a sequence of smooth diffeomorphisms $\psi_{l}: \bar{\Sigma} \rightarrow \overline{\Sigma_{l}}$, which in the $C^{\infty}$ sense converges to the identity map. We define

$$
J_{l}=\left(D \psi_{l}\right)^{-1} \circ J \circ D \psi_{l}
$$

Then $\left\{J_{l}\right\}_{l \in \mathbb{N}}$ is a sequence of complex structures on $\Sigma$ which $C^{\infty}$ converges to $J$.

For every $l$ we define $f_{l}=f_{0} \circ \psi_{l}$ a smooth up to the boundary holomorphic function on $\left(\Sigma, J_{l}\right)$. Let $n \in \mathbb{N}$ be fixed. Since the graph of $f_{0}$ belongs to $\widehat{T}$, we get that there exists $l_{0} \in \mathbb{N}$ so that for every $l>l_{0}$ we have

$$
f_{l}(z) \in \operatorname{Int}\left(\gamma_{z}^{n}\right) \quad(z \in \partial \Sigma) .
$$

From [12] it follows that there exists a sequence $\left\{g_{l}\right\}_{l \geq l_{0}}$ of $C^{k, \alpha}$ functions on $\bar{\Sigma}$ such that

a) $g_{l}$ is holomorphic on $\left(\Sigma, J_{l}\right)$.

b) $g_{l}$ solves the Riemann-Hilbert problem on $\left(\Sigma, J_{l}\right)$ for $\left\{\gamma_{z}^{n}\right\}_{z \in \partial \Sigma}$.

c) The winding number of the outer normal to $\gamma_{z}^{n}$ at $g_{l}(z)$ along $\partial \Sigma$ is at most $2 g+m-1$.

Let $l \rightarrow \infty$. Using Gromov's compactness theorem we get that there exists a $C^{k, \alpha}(\Sigma)$ holomorphic function $\widetilde{f}_{n}$ on $(\Sigma, J)$ such that

a) $\widetilde{f}_{n}$ solves the Riemann-Hilbert problem on $\Sigma$ for $\left\{\gamma_{z}^{n}\right\}_{z \in \partial \Sigma}$.

b) The winding number of the outer normal to $\gamma_{z}^{n}$ at $\widetilde{f}_{n}(z)$ along $\partial \Sigma$ is at most $2 g+m-1$.

Let $n \rightarrow \infty$, and the proof is finished by the compactness theorem.

\section{REFERENCES}

1. L.Ahlfors, Bounded analytic functions, Duke Math. J. 14 (1947), 1-11. MR0021108 (9:24a)

2. L.Ahlfors, Open Riemann surfaces and extremal problems on compact subregions, Comment. Math. Helv. 24 (1950), 100-134. MR0036318 (12:90b)

3. H.Alexander and J.Wermer, Polynomial hulls with convex fibers, Math. Ann. 266 (1981), 243-257. 
4. D.E.Barrett, Failure of averaging on multiply connected domains, Ann. Inst. Fourier 40 (1990), 357-370. MR:1070831 (92b:30050)

5. H.Begehr and M.A.Efendiev, On the asymptotics of meromorphic solutions for nonlinear Riemann-Hilbert problems, Math. Proc. Cambridge Philos. Soc. 127 (1999), 159-172. MR.1692479 (2000c:30076)

6. S.R.Bell, Finitely generated function fields and complexity in potential theory in the plane, Duke Math. J. 98 (1999), 187-207. MR.1687563(2000i:30015)

7. S.R.Bell, Ahlfors maps, the double of a domain, and complexity in potential theory and conformal mapping, J. Anal. Math. 78 (1999), 329-344. MR.1714417 (2000m:30012)

8. S.R.Bell, A Riemann surface attached to domains in the plane and complexity in potential theory, Houston J. Math. 26 (2000), 277-297. MR.1814239 (2001m:30009)

9. S.R.Bell, Complexity in complex analysis, Adv. Math. 172 (2002), 15-52. MR1943900 (2003m:30016)

10. E.Bishop, Subalgebras of functions on a Riemann surface, Pacific J. Math. 8 (1958), 29-50. MR0096818 (20:3300)

11. Y.-B.Chung, Higher order extremal problem and proper holomorphic mapping, Houston J. Math. 27 (2001), 707-718. MR1864806 (2002j:30031)

12. M.Černe, Nonlinear Riemann-Hilbert problem for bordered Riemann surfaces, Amer. J. Math. 126 (2004), 65-85. MR.2033564 (2004k:30092)

13. M.Černe and F.Forstnerič, Embedding some bordered Riemann surfaces in the affine plane, Math. Res. Lett. 9 (2002), 683-696. MR.1906070 (2003j:32021)

14. M.Černe and J.Globevnik, On holomorphic embedding of planar domains into $\mathbb{C}^{2}$, J. Anal. Math. 81 (2000), 269-282. MR1785284 (2001g:32047)

15. E.M.Čirka, Regularity of boundaries of analytic sets (Russian) Math. Sb. (NS) 117 (1982), 291-334. MR0648411 (83f:32009)

16. J.Diller Failure of weak holomorphic averaging on multiple connected domains, Math. Z. 217 (1994), 167-177. MR.1296392 (95m:30034)

17. M.A.Efendiev and W.L.Wendland, Nonlinear Riemann-Hilbert problems for multiply connected domains, Nonlinear Anal. 27 (1996), 37-58. MR1390711 (97h:30057)

18. M.A.Efendiev and W.L.Wendland, Nonlinear Riemann-Hilbert problems without transversality, Math. Nachr. 183 (1997), 73-89. MR1434976 (98b:30038)

19. M.A.Efendiev and W.L.Wendland, Nonlinear Riemann-Hilbert problems for doubly connected domains and closed boundary data, Topol. Methods Nonlinear Anal. 17 (2001), 111-124. MR.1846981 (2002d:30045)

20. F.Forstnerič, Polynomial hulls of sets fibered over the circle, Indiana Univ. Math. J. 37 (1988), 869-889. MR0982834 (90g:32018)

21. M.Gromov, Pseudo-holomorphic curves in symplectic manifolds, Invent. Math. 81 (1985), 307-347.

22. C.D.Hill and G.Taiani, Families of analytic discs in $\mathbb{C}^{n}$ with boundaries on a prescribed CR submanifold, Ann. Scuola. Norm. Sup. Pisa 5 (1978), 327-380. MR0501906 (80c:32023)

23. L.K.Kodama, Boundary measures of analytic differentials and uniform approximation on a Riemann surface, Pacific J. Math. 15 (1965), 1261-1277. MR0190327|(32:7740)

24. W.Koppelman, The Riemann-Hilbert problem for finite Riemann surfaces, Comm. Pure Appl. Math. 12 (1959), 13-35. MR0146394 (26:3916)

25. F.G.Maksudov, M.A.Efendiev, The nonlinear Hilbert problem for a doubly connected domain (Russian), Dokl. Akad. Nauk SSSR 290 (1986), 789-791. MR0863355 (88j:30088)

26. P.Pansu, Compactness, in M.Audin and J.Lafontaine, Editors, Holomorphic curves in symplectic geometry, Progress in Mathematics 117, Birkhäuser (1994), 233-249. MR1274932

27. E.A.Poletskiur, The Euler-Lagrange equations for extremal holomorphic mappings of the unit disk, Michigan Math. J. 30 (1983), 317-333. MR0725784 (85b:32038)

28. M.Schiffer and D.C.Spencer, Functionals on finite Riemann surfaces, Princeton Univ. Press., Princeton, 1954. MR0065652(16:461g)

29. Z.Slodkowski, Polynomial hulls in $\mathbb{C}^{2}$ and quasicircles, Ann. Scuola Norm. Sup. Pisa 16 (1989), 367-391. MR:1050332 (91m:32016)

30. A.I.Šnirelman, The degree of a quasiruled mapping and a nonlinear Hilbert problem (Russian) Mat. Sb. 18 (1972), 373-396. MR0326521 (48:4865) 
31. E.Wegert, Nonlinear boundary value problems for holomorphic functions and singular integral equations, Mathematical Research 65, Akademie-Verlag, Berlin, 1992. MR 1206907 (94b:30049)

32. R.Ye, Gromov's compactness theorem for pseudo holomorphic curves, Trans. Amer. Math. Soc. 342 (1994), 671-694. MR.1176088(94f:58030)

Department of Mathematics, University of Ljubljana, Jadranska 19, 1111 Ljubljana, Slovenia

E-mail address: miran.cerne@fmf.uni-lj.si

Department of Mathematics, University of La Laguna, 38771 La Laguna, Tenerife, SPAIN

E-mail address: mflores@ull.es 